\title{
Collision Avoidance and Dynamic Sleep Scheduling Technique for Cluster Based Underwater Wireless Sensor Networks (UWSN)
}

D. Anuradha ( $\sim$ anuradha123phd@gmail.com )

Panimalar Engineering College

S. Suresh

SRM Institute of Science and Technology

P. Muneeshwari

SRM Institute of Science and Technology

\section{Research Article}

Keywords: UWSN, Sink, Collision, Sleep Scheduling, Cluster

Posted Date: July 20th, 2021

DOl: https://doi.org/10.21203/rs.3.rs-714850/v1

License: (c) (i) This work is licensed under a Creative Commons Attribution 4.0 International License.

Read Full License 


\title{
Collision Avoidance and Dynamic Sleep Scheduling Technique for Cluster Based Underwater Wireless Sensor Networks (UWSN)
}

\author{
D.Anuradha \\ Professor ,Department of Computer Science and Business Systems,Panimalar Engineering College \\ anuradha123phd@gmail.com \\ Dr.S.Suresh, \\ Assistant Professor(SL.G),Department of CSE, SRM Institute of science and Technology, Ramapuram \\ campus,Chennai \\ sureshs12@srmist.edu.in \\ Dr.P.Muneeshwari \\ Assistant Professor(SL.G),Department of CSE, SRM institute of Science and Technology,Ramapuram \\ Campus,Chennai \\ muneeswp@srmist.edu.in
}

\begin{abstract}
In UWSN, during clustering, there may be occurrence of intra cluster collision. In order to overcome this issue, in this paper, we propose to design protocol to efficiently handle the intra-cluster collisions and to design sleep-wake up scheduling scheme for the data aggregation. In this case, the cluster head coordinates with its cluster members to transmit (append) their data packets with partially overlapping transmission times. After the $\mathrm{CH}$ finishes transmitting its packets to the courier nodes, it starts to receive incoming appended packets from its members. It then sends the packet to its parent $\mathrm{CH}$ towards the sink applying data fusion and sending the aggregated packet in TDMA period based on the receiver oriented sleep scheduling scheme. By simulation results, we show that the proposed technique minimizes collision and transmission delay.
\end{abstract}

Keywords: UWSN, Sink, Collision, Sleep Scheduling, Cluster

\section{Introduction}

\subsection{UWSN}

Since many years Underwater Wireless Sensor Network (UWSN) have proved its strength of utilization in various applications of monitoring, exploration, surveillance, attack protection, and tracking in harsh underwater environment ${ }^{[1]}$. The underwater channel is characterized by prolonged propagation times and frequencydependent attenuation that is highly affected by the distance between deployed nodes as well as by the link orientation. UWSN consists of a number of nodes that processes the data and transmit it to other nodes or sink ${ }^{[2]}$. Since a radio signal does not work under the water due to high attenuation therefore acoustic signals are used in underwater networks. However in comparison to radio channels, acoustic channels exhibit high error rates, low available bandwidth and long propagation delays. Cluster-based UWSNs have been investigated by researchers to achieve the network scalability and efficiency, which maximize network lifetime and reduce bandwidth consumption by using local collaboration among sensor nodes.

The application of UWSN has huge potential for environment such as environmental monitoring, undersea explorations, mine reconnaissance, disaster prevention, distributed tactical surveillance, oceanographic data collection, target detection and underwater military applications ${ }^{[3]}$.

\subsection{Collision Avoidance in UWSN}

Random deployment of sensor nodes inevitably triggers collisions during message exchanges ${ }^{[4]}$. In a cluster based data aggregation, the cluster members should transmit the collected data to the cluster head in a collision 
free manner with reduced power consumption. Hence efficient scheduling techniques should be applied while aggregating data at the cluster head.

Scheduling mechanisms control the transmission process by indicating which packets must be transmitted and which packets must be dropped. The main objectives of scheduling techniques are to reduce the collision among the flows and conserve energy. TDMA based scheduling techniques aim to reduce the collision by assigning different slots to the nodes.

In this paper, Collision Avoidance and Dynamic Sleep Scheduling Technique is proposed to efficiently handle the intra-cluster collisions and sleep-wake up scheduling

\section{Related Works}

Wen-Hwa Liao et al. ${ }^{[2]}$ have proposed a receiver-initiated MAC protocol with packet train for UWSN called multi-receiver MAC protocol (MR-MAC). The protocol will increase the throughput and reduce of the transmission of control packet for handshaking and their MRMAC protocol could make more than two nodes to communicate in one handshake held by a main receiver. By scheduling the packet transmission time, the data packet would be sent in a packet train manner and the receiver could receive data packet without collision.

Stefano Basagni et al. ${ }^{[5]}$ have investigated the effect of packet size selection on the performance of media access control (MAC) protocols for underwater wireless sensor networks, namely, carrier sense multiple access (CSMA) and the distance-aware collision avoidance protocol (DACAP). The metrics such as throughput efficiency (the ratio between the delivered bit rate and the offered bit rate), end-to-end packet latency, measured "per meter" to allow for different sizes of deployment areas, and the energy consumed to correctly deliver an information bit to the network collection point were estimated.

Zuodong $\mathrm{Wu}$ et al ${ }^{[7]}$ have proposed a minimum-latency aggregation scheduling scheme for minimizing the data aggregation time using a Shortest Hop Tree based algorithm in tree construction phase together with its theoretical bound .It have used the concept of Virtual Slot to allow overlap communications with different time slots by applying layer by layer property that making the multiplexing opportunities of the time domain scheme easy to understand and implement. However, when reserving the channel there may be chances for conflict due to overlap communications at sink node.

Tayseer Alkhdour et al. ${ }^{[8]}$ have developed energy-efficient distributed schedule-based protocol to build an energy efficient routing tree and TDMA scheduling framework. This model is solved for different network configurations. However, additional cost functions such as delay are not considered here.

Kurtis Kredo et al ${ }^{[10]}$ have proposed the Staggered TDMA Underwater MAC Protocol (STUMP) utilizing node position diversity through estimation of node propagation delay information to schedule overlapping transmissions without conflicts for collision free when channel reservation and also for reducing schedule overhead by using logical rings surrounding each node which schedules each node to transmit once per frame for each transmission by reserving all the rings. The packets from two different nodes reach successfully without collision even if the packets were transmitted at the same time. It can cause delay since each node transmits only once per frame. When using TDMA in UWSN, it is difficult to attain synchronization among all network nodes due to variable propagation delays

\section{Proposed Solution}

\subsection{Overview}

In this paper, we propose to design protocol to efficiently handle the intra-cluster collisions and to design sleepwake up scheduling scheme for the data aggregation. In this case, the cluster head coordinates with its cluster members to transmit (append) their data packets with partially overlapping transmission times. After the $\mathrm{CH}$ finishes transmitting its packets to the courier nodes, it starts to receive incoming appended packets from its members. It then sends the packet to its parent $\mathrm{CH}$ towards the sink applying data fusion and sending the aggregated packet in TDMA period based on the receiver oriented sleep scheduling scheme.

\subsection{System model}


EDETA (Energy-efficient aDaptive hiErarchical and robusT Architecture) is a routing protocol. It is a hierarchical protocol by which the nodes arrange themselves in clusters with one of them acting as a clusterhead $(\mathrm{CH})$. The $\mathrm{CHs}$ form a ranking structure between themselves in order to send the gathered and aggregated data from the other nodes to the sink in a multi-hop manner. This EDETA protocol supports more than one sink providing more scalability.

Courier nodes are organized in this protocol so as to collect the aggregated data from the selected $\mathrm{CH}$. These nodes to make the $\mathrm{CH}$ aware of its presence broadcast the HELLO messages and inter cluster communication is enabled in order to forward the sensed data. (Refer paper 1 for more details)

Thus to avoid, intra-cluster collisions, single-channel handshaking-based MAC protocol based on reverse opportunistic packet appending (ROPA) ${ }^{[10]}$ is applied.

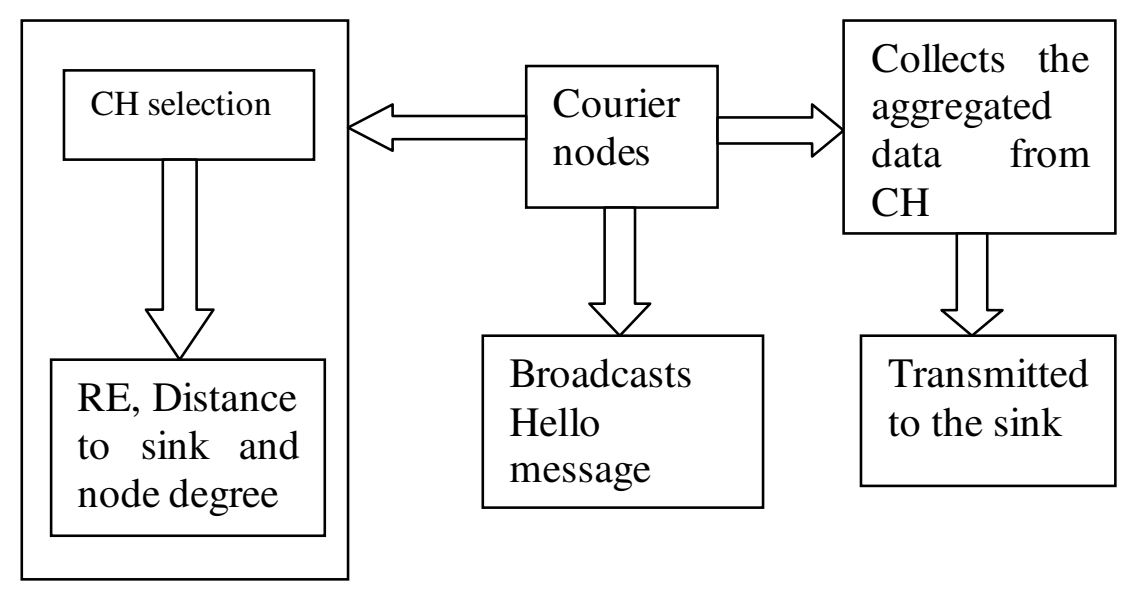

Figure 1 Block Diagram

\subsubsection{Cluster Formation and Courier Node Deployment}

Initially the nodes arrange themselves in clusters with one of them acting as a cluster-head $(\mathrm{CH})$. The $\mathrm{CH}$ selection is based upon the residual energy, distance to sink and the node degree.

The $\mathrm{CH}$ election is based on a random number which should be lesser than the threshold value $T H(n)$,

$$
T H(n)=\frac{c}{|\mathrm{~N}|-2 c} \times \alpha, n \in \mathrm{N}
$$

where $c$ - Number of clusters,

$$
\begin{aligned}
& \mathrm{N} \text { - set of nodes, } \\
& \alpha \text { - parameter which depends on time }
\end{aligned}
$$

If the number randomly generated is lower than the calculated threshold, a node can become a $\mathrm{CH}$ only if its remaining energy $\varepsilon$ is greater than value $\varepsilon(n)$.

$$
\varepsilon(n)=\varepsilon_{a} \times \frac{2 E_{R}}{2 E_{R}+D_{S} d_{i}}, n \in \mathrm{N}
$$


where $E_{R}$ is the residual energy of each node, $\mathrm{D}_{\mathrm{S}}$ is the distance to sink and $d_{i}$ is the node degree.

The Courier nodes are deployed into the clusters for collecting the aggregated data from the $\mathrm{CH}$. The courier nodes after reaching any specified position will broadcast HELLO packets so that, ordinary nodes around it can know about their presence. Then ordinary nodes will forward the sensed data within a specified amount of time, which is defined in the HELLO packet.

\section{HELLO packet format}

HELLO packet is used for the advertisement of the courier nodes. It consists of four fields, NodeID, Expiry Time, HopID and Max Hop Count.

\begin{tabular}{|l|l|l|l|}
\hline NodeID & Expiry Time & HopID & Max Hop Count \\
\hline & & & \\
\hline
\end{tabular}

Table-1 HELLO Packet format

\section{Algorithm}

1. Courier nodes $\mathrm{CR}_{\mathrm{i}}$ are deployed for collecting data from the CHs.

2. $\mathrm{CR}_{\mathrm{i}}$ broadcasts HELLO message on reaching a particular position.

3. If any $\mathrm{CR}_{\mathrm{p}}$ receives this packet, then

4. $\quad \mathrm{CR}_{\mathrm{p}}$ gets the HopID $\mathrm{N}_{\mathrm{r}}$ received from the HEELO packet

5. If $\mathrm{N}_{\mathrm{r}}$ is less than $\mathrm{N}_{\mathrm{p}}$, then

6. HELLO packet is discarded.

7. Else

8. $\quad \mathrm{p}=\mathrm{r}$.

9. End if

10. If MaxHopCount $>4$ then

11. HELLO packet is broadcast again

12. Else

13. it is discarded.

14. End if

15. End if

Figure 3 shows the deployment of courier node and data forwarding along clusters.

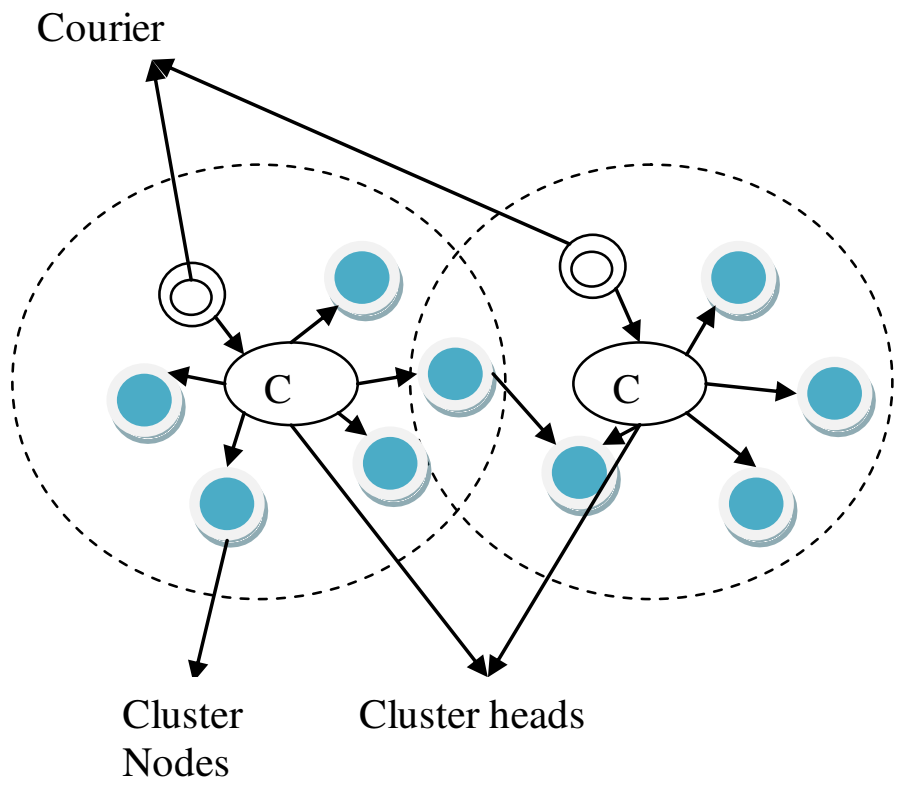

Figure 2 Deployment of courier nodes with intercluster routing 


\subsection{Collision Avoidance Algorithm}

The steps involved in this technique are as follows:

1. $\mathrm{CH}$ broadcasts the RTS packet to its cluster members $\left(\mathrm{CM}_{\mathrm{i}}\right)$.

\begin{tabular}{|c|l|l|l|}
\hline $\begin{array}{c}p \\
\mathrm{~N}^{i}\end{array}$ & $\mathrm{~N}^{\max }$ & $\mathrm{T}_{\text {busy,RTS }}$ & $\mathrm{t}_{\mathrm{cf}}$ \\
\hline & & & \\
\hline
\end{tabular}

Table 2 Format of RTS Packet

The format of RTS packet is shown in Table- 2 .

Here,

$N_{i}^{p}$ denotes total number of primary data packets to be transmitted

$N_{\max }^{S}$ denotes maximum permitted number of secondary data packets that can be appended for the current handshake.

$T_{\text {busy,RTS }}$ denotes expected busy duration

$t_{c f}$ denotes collision free RTA schedule computed for its neighbors.

2. Upon overhearing an RTS packet, each potential $\mathrm{CM}_{\mathrm{i}}$ verifies whether it has any data packets to transmit.

3. If packet exists, then it generates the request to append (RTA) packet with the 3-tuple, $\left[\mathrm{N}^{\text {rel }}, N^{\text {new }}, T_{\text {busy,RTA }}\right]$

where $\mathrm{N}^{\text {rel }}{ }_{\mathrm{i}}$ and $\mathrm{N}^{\text {new }}{ }_{\mathrm{j}}=$ number of relayed and new packets that is wishes to append respectively.

$\mathrm{N}^{\mathrm{rel}}{ }_{\mathrm{i}}+\mathrm{N}^{\text {new }}{ }_{\mathrm{j}} \leq \mathrm{N}_{\text {max }}^{S}$

4. Before responding with RTA, each $\mathrm{CM}_{\mathrm{i}}$ verifies the collision free schedule provided by the RTS packet.

5. If the schedule does not require the $\mathrm{CM}_{\mathrm{i}}$ to defer its RTA transmission, it performs the transmission immediately. Or else it defers it RTA transmission by the amount of time indicated within the schedule.

6. The intended $\mathrm{CM}_{\mathrm{i}}$ upon receiving an RTS packet verifies whether it has any data packets in return before responding with clear to send (CTS) packet. Unlike the RTA response, the receiver can transmit its CTS without any deferment.

7. When the $\mathrm{CM}_{\mathrm{i}}$ wishes to append packets, it specifies $\mathrm{N}_{\mathrm{R}}^{\mathrm{rel}}$ and $\mathrm{N}^{\text {new }}{ }_{\mathrm{R}}$ in its $\mathrm{CTS}$ reply.

$$
\mathrm{N}^{\mathrm{rel}}{ }_{\mathrm{R}}+\mathrm{N}^{\text {new }}{ }_{\mathrm{R}} \leq \mathrm{N}_{\text {max }}^{S} \text {. }
$$


8. When the $\mathrm{CM}_{\mathrm{i}}$ does not have any packet to append, the $\mathrm{N}_{\mathrm{R}}^{\text {rel }}$ and $\mathrm{N}^{\text {new }}{ }_{\mathrm{R}}$ are set to zero.

9. After broadcasting an RTS packet, $\mathrm{CH}_{\mathrm{i}}$ waits until a reference time $\mathrm{T}_{\text {ref }}$, that is sufficiently long to accommodate even its most distant first hop neighbors incoming RTA packet.

10. Having acquired all incoming requests, $\mathrm{CH}_{\mathrm{i}}$ allocates its available secondary data slots, $\mathrm{N}_{\max }^{S}$ using a simple strategy that prioritizes all relayed packets over new packets.

11. A CTA packet is then broadcasted to inform they should append.

12. For each $\mathrm{CM}_{\mathrm{i}}$, the CTA carries the following 4-tuple:

[node ID, $\mathrm{N}_{\mathrm{G}}^{\mathrm{rel}}+\mathrm{N}_{\mathrm{G}}^{\text {new }}$, dwait,i]

where $\mathrm{N}_{\mathrm{G}}^{\mathrm{rel}}$ and $\mathrm{N}_{\mathrm{G}}^{\text {new }}=$ number of relayed and new packets that appender node $\mathrm{i}$ can transmit respectively.

$\mathrm{d}_{\mathrm{wait}, \mathrm{i}}=$ duration that node $\mathrm{i}$ must wait before it can start to append its packets.

Similarly to other control packets, the CTA also contains a busy duration field,

13. Immediately after transmitting the $\mathrm{CTA}, \mathrm{CH}_{\mathrm{i}}$ starts transmitting its primary packets to its intended receiver.

14. After the $\mathrm{CH}$ finishes transmitting its packets to the courier nodes, it starts to receive incoming appended packets from its members that arrive in a collision-free packet train.

15. Each $\mathrm{CM}_{\mathrm{i}}$ verifies its previous request has been granted by looking up the grant decisions in the CTA packet.

16. A non-granted $\mathrm{CM}_{\mathrm{i}}$ remains silent for duration of $\mathrm{d}_{\mathrm{CTA} \text {, silent }}$.

17. Each granted $\mathrm{CM}_{\mathrm{i}}$ start to transmit its granted number of secondary data packets after the specified waiting duration to append has been passed.

18. After transmitting its share of appended packets, it remains silent so that it does not disrupt the $\mathrm{CH}^{\text {'s }}$ reception of packets from other $\mathrm{CM}_{\mathrm{i}}$ 's.

The sleep cycle is computed in the next section.

\subsection{Dynamic Sleep Scheduling}


Each cluster member $\left(\mathrm{CM}_{\mathrm{i}}\right)$ includes working and sleeping period. The sleep schedule is applied from a lower level $\mathrm{CH}$ to higher level $\mathrm{CH}$. That is, the $\mathrm{CH}$ acts as a receiver which receives packets from its members and send to its parent $\mathrm{CH}$ towards the sink. It applies data fusion and sends the aggregated packet in TDMA period.

The steps involved in this process are as follows:

1. During $\mathrm{t}_{\text {start }}\left(0 \leq t_{\text {start }} \leq T\right)$, the node begins to work in the first TDMA period.

2. The sink node first selects it $\mathrm{t}_{\text {start }}$ and decides the transmission sequence of its clusters.

3. The sink node broadcasts its decision to its clusters.

4. Each $\mathrm{CH}$ computes its own start moment $\mathrm{t}_{\text {start }}$ as follows:

$$
\mathrm{t}_{\mathrm{start}}(\mathrm{CM})=\left\{\mathrm{T}+\mathrm{t}_{\mathrm{start}}(\mathrm{CH})+\left[\mathrm{Y}_{\mathrm{CH}}-\mathrm{Z}(\mathrm{CH})-1\right] \mathrm{T}_{\mathrm{p}}-\mathrm{Q}_{\mathrm{CH}}(\mathrm{CM}) \bmod (\mathrm{T})\right.
$$

where $\mathrm{Y}_{\mathrm{CH}}=$ serial number of $\mathrm{CH}$ in the receiving sequence of $\mathrm{CM}$

$\mathrm{Z}(\mathrm{CH})=$ number of child node of $\mathrm{CH}$

$\mathrm{Q}_{\mathrm{CH}}=$ link delay.

5. Each $\mathrm{CH}$ broadcasts its decision to its own cluster members. This procedure is repeated until reaching the leaf nodes.

6. The transmitting order of cluster member is decided by the distance to the $\mathrm{CH}$, where each node sorts the order of transmission from its cluster members by descending values of the distance from the children.

7. As per (1), $t_{\text {start }}$ of each node is decided by multiple factors, including the number of its cluster members, the position in transmission sequence E, the propagation delay to its father node Q, and the starting moment of its father node.

8. After $\mathrm{t}_{\mathrm{start}}(\mathrm{CH})$ has been decided, when transmissions begin, the start time of each period of node $\mathrm{m}$ is:

$$
\mathrm{t}_{\mathrm{start}}(\mathrm{CH})+\mathrm{nT}\{\mathrm{n}=0,1,2, \ldots\}
$$

9. $\mathrm{CH}$ then applies data fusion and send the aggregated packet in TDMA period.

\section{Simulation Results}

\subsection{Simulation Parameters}

We use NS2 ${ }^{[12]}$ to simulate our proposed Collision Avoidance and Dynamic Sleep Scheduling (CADSS) Technique. The simulation parameters are shown in Table 1

Table 1: Simulation parameters

\begin{tabular}{|l|l|}
\hline Number of Nodes & $50,100,150$ and 200 \\
\hline Area & 1000 X 1000 \\
\hline MAC & Undrwater Mac \\
\hline Simulation Time & $50 \mathrm{sec}$ \\
\hline Traffic Source & CBR \\
\hline Interval & $100,200,300,400$ and 500 \\
\hline Propagation & TwoRayGround \\
\hline
\end{tabular}




\begin{tabular}{|l|l|}
\hline Antenna & Omni Antenna \\
\hline Initial Energy & $10000 \mathrm{~J}$ \\
\hline Transmission Power & 2.0 \\
\hline Receiving Power & 0.75 \\
\hline
\end{tabular}

\subsection{Performance Metrics}

We evaluate performance of the new protocol mainly according to the following parameters. We compare the reverse opportunistic packet appending (ROPA) ${ }^{[10]}$ protocol with our proposed CADSS protocol.

Average Packet Delivery Ratio: It is the ratio of the number of packets received successfully and the total number of packets transmitted.

Packet Drop: It is the number of packets dropped during the data transmission

Energy Consumption: It is the amount of energy consumed by the nodes to transmit the packets to the destination.

\subsection{Results \& Analysis}

The simulation results are presented in the next section.

\section{A. Based on Nodes} traffic.

In our first experiment we are varying the number of nodes as 50,100,150 and 200 for CBR

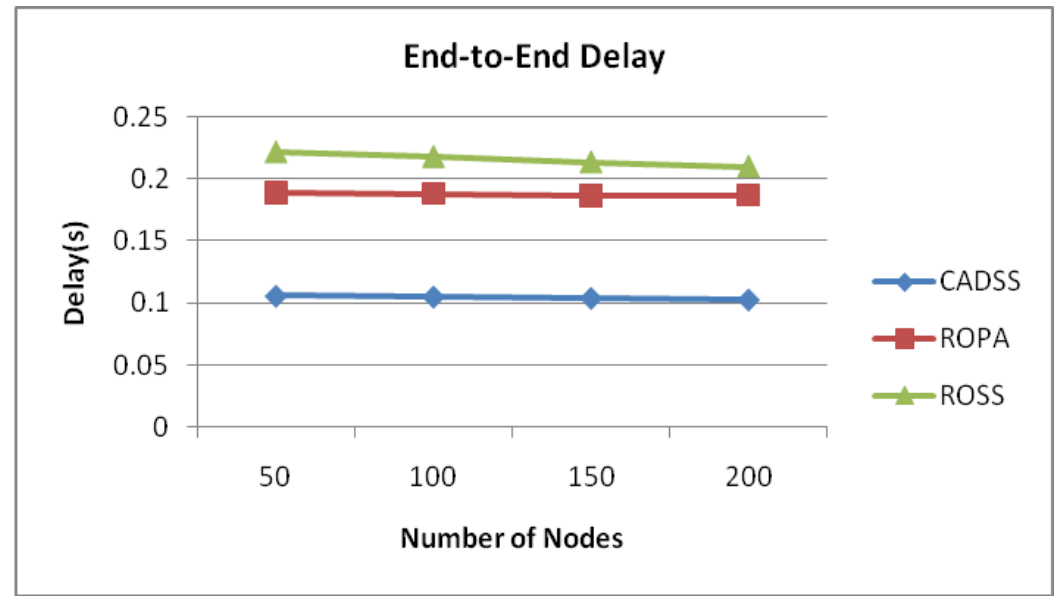

Fig 3: Delay for varying nodes

Figure 3 shows the delay occurred for CADSS, ROPA and ROSS techniques when the number of nodes is varied. The increase in nodes results in decrease in delay. As seen from the figure, the delay of CADSS decreases from 0.105 to $0.102 \mathrm{~s}$, the delay of ROPA decreases from 0.188 to $0.186 \mathrm{~s}$ and the delay of ROSS decreases from 0.221 to 0.209 s. However CADSS has $44 \%$ lesser delay ratio than ROPA and $51 \%$ lesser than ROSS technique. 


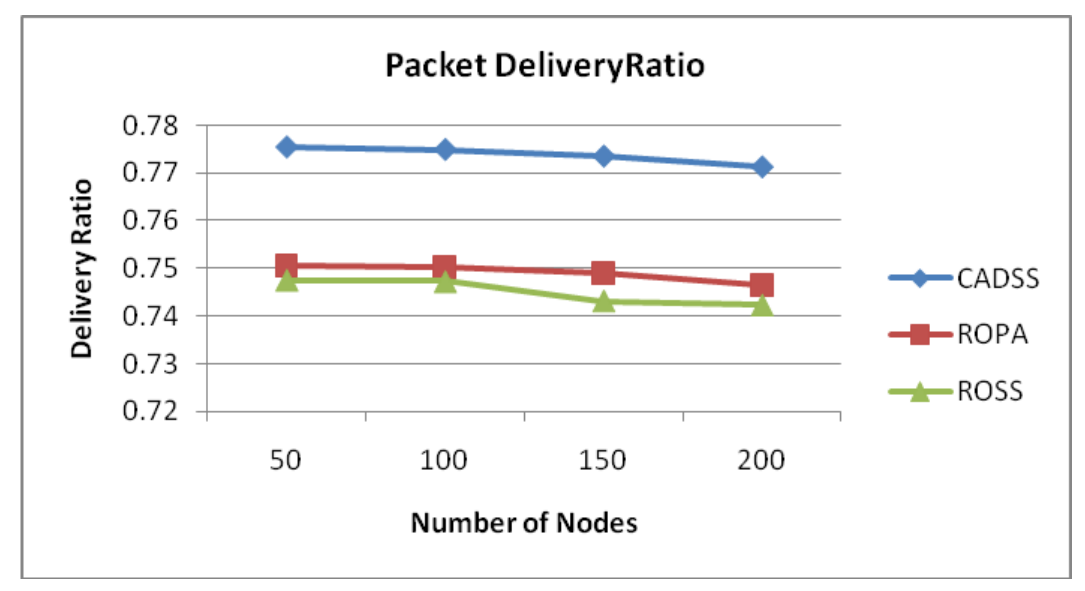

Fig 4 Delivery Ratio for varying nodes

Figure 4 shows the delivery ratio occurred for CADSS, ROPA and ROSS techniques when the number of nodes is varied. The increase in nodes results in decrease in packet delivery ratio. As seen from the figure, the delivery ratio of CADSS decreases from 0.7754 to 0.7712 , the delivery ratio of ROPA decreases from 0.7505 to 0.7464 and the delivery ratio of ROSS decreases from 0.7475 to 0.7422 . Hence the delivery ratio of CADSS is $3.1 \%$ higher than ROPA and 3.7\% higher than ROSS.

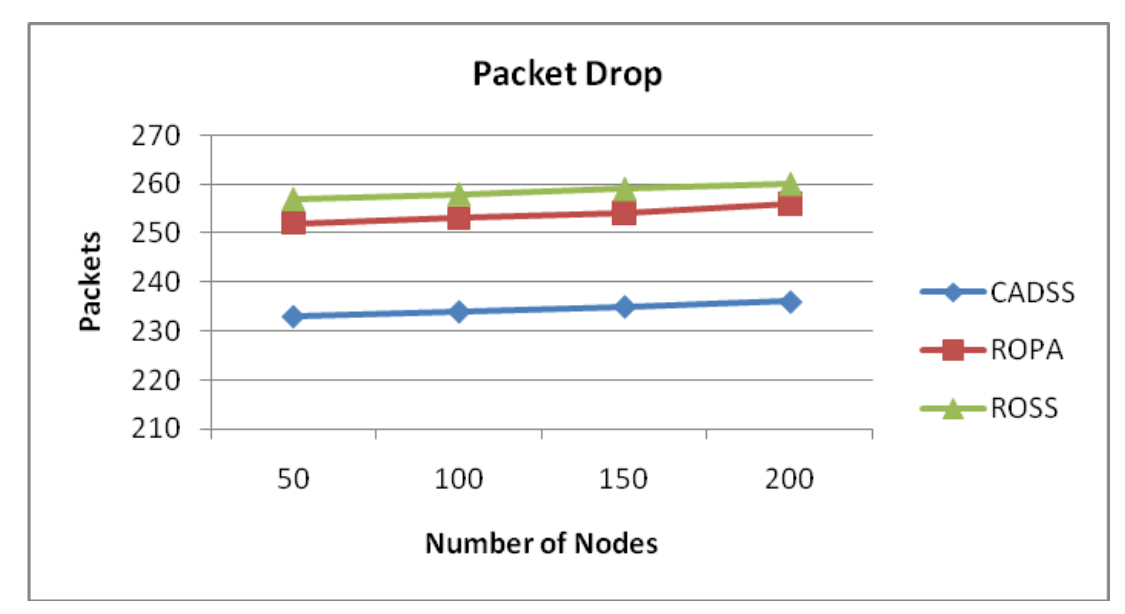

Fig 5 Packet Drop for varying nodes

Figure 5 shows the packet drop occurred for CADSS, ROPA and ROSS techniques when the number of nodes is varied. The increase in nodes results in increase in packet drop. As seen from the figure, the drop of CADSS increased from 233 to 236, the drop of ROPA increases from 252 to 256 and the drop of ROSS increases from 257 to 260 . Hence CADSS has 7.5\% lesser packet drop than ROPA and 9.2\% lesser packet drop than ROSS. 


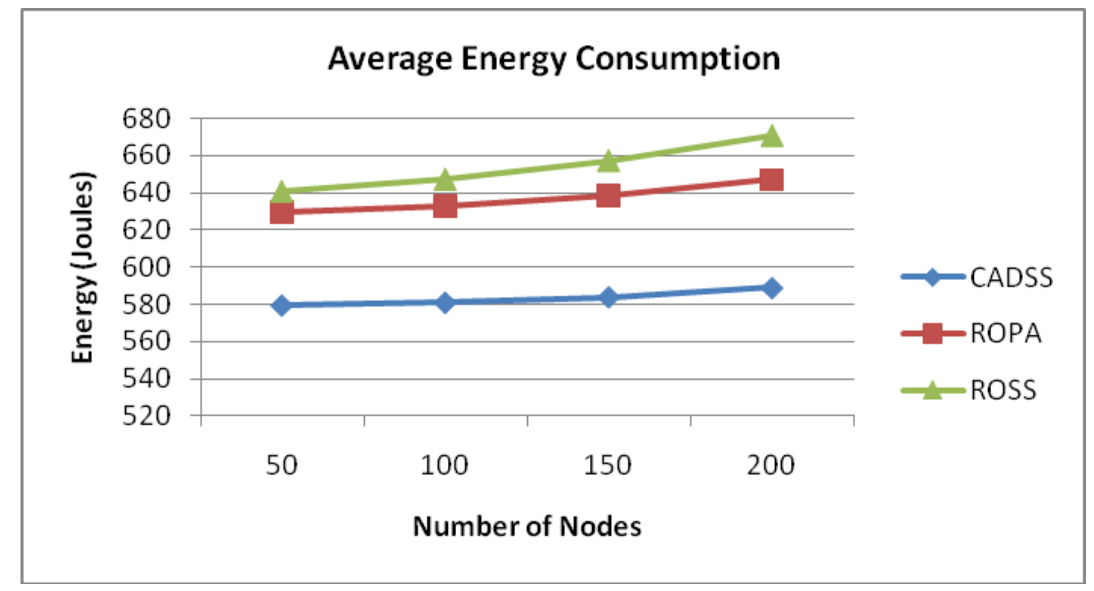

Fig 6 Energy Consumption for varying nodes

Figure 6 shows the average energy consumption occurred for CADSS, ROPA and ROSS techniques when the number of nodes is varied. The increase in nodes results in increase in energy consumption. As seen from the figure, the energy consumption of CADSS increased from 579.5 to 588.9 , the energy consumption of ROPA increases from 629.3 to 646.8 and the energy consumption of ROSS increases from 640.4 to 670.4. Hence CADSS has $8 \%$ lesser energy consumption than ROPA and $10 \%$ lesser energy consumption than ROSS.

\section{B. Based on Interval}

Next, the cluster formation timer interval is varied as $100,200,300,400$ and 500 seconds.

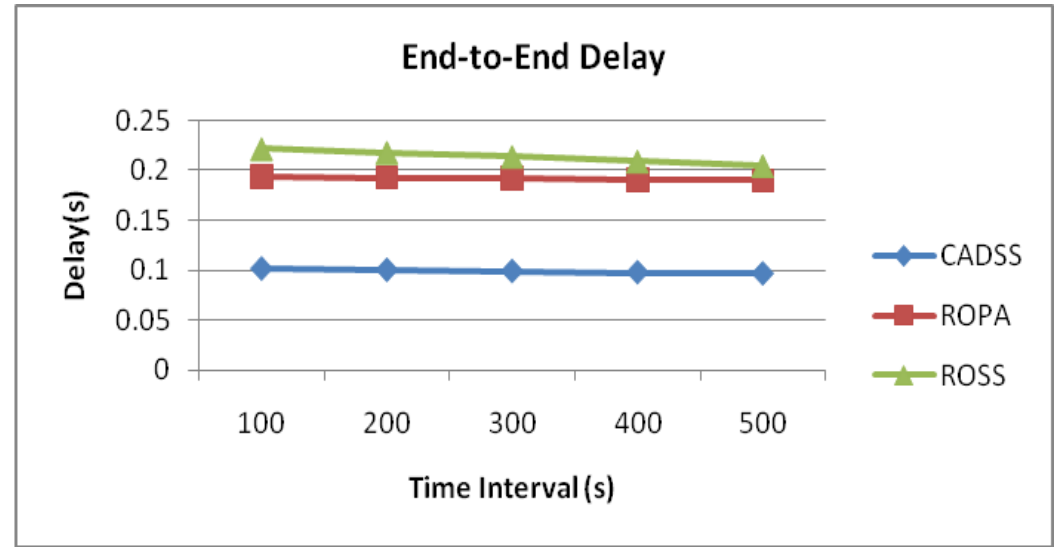

Fig 7 Delay for varying Time

Figure 7 shows the delay occurred for CADSS, ROPA and ROSS techniques when the interval is varied. The increase in interval results in decrease in delay. As seen from the figure, the delay of CADSS decreases from 0.101 to $0.096 \mathrm{~s}$, the delay of ROPA decreases from 0.193 to $0.186 \mathrm{~s}$ and the delay of ROSS decreases from 0.221 to $0.204 \mathrm{~s}$. Hence CADSS has $48 \%$ lesser delay than ROPA and $53 \%$ lesser delay than ROSS. 


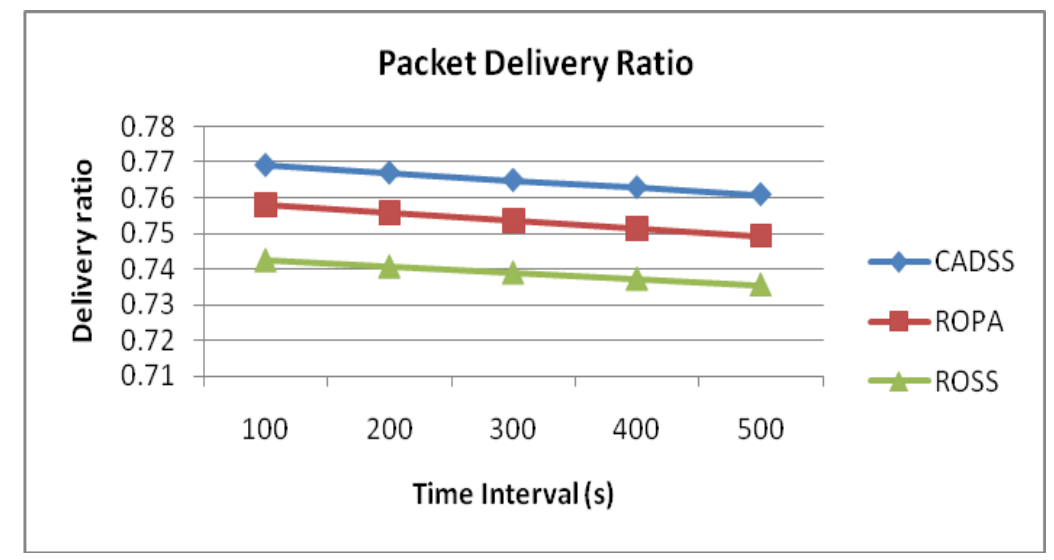

Fig 8 Delivery Ratio for varying Time

Figure 8 shows the delivery ratio occurred for CADSS, ROPA and ROSS techniques when the interval is varied. The increase in interval results in decrease in packet delivery ratio. As seen from the figure, the delivery ratio of CADSS decreased from 0.769 to 0.760 , the delivery ratio of ROPA decreases from 0.757 to 0.749 and the delivery ratio of ROSS decreases from 0.0742 to 0.735 . Hence CADSS has $1.4 \%$ higher delivery ratio than ROPA and $3.3 \%$ higher delivery ratio than ROSS.

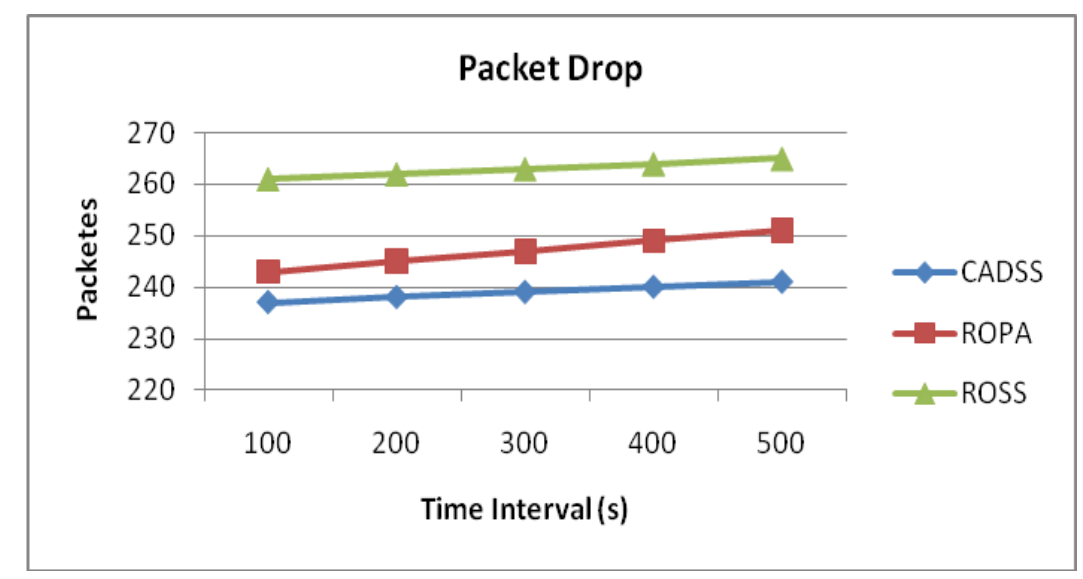

Fig 9 Packet Drop for varying Time

Figure 9 shows the packet drop occurred for CADSS, ROPA and ROSS techniques when the interval is varied. The increase in interval results in increase in packet drop. As seen from the figure, the drop of CADSS increased from 237 to 241, the drop of ROPA increases from 243 to 251 and the drop of ROSS increases from 261 to 265. Hence CADSS has $3 \%$ lesser packet drop than ROPA and 9\% lesser packet drop than ROSS.

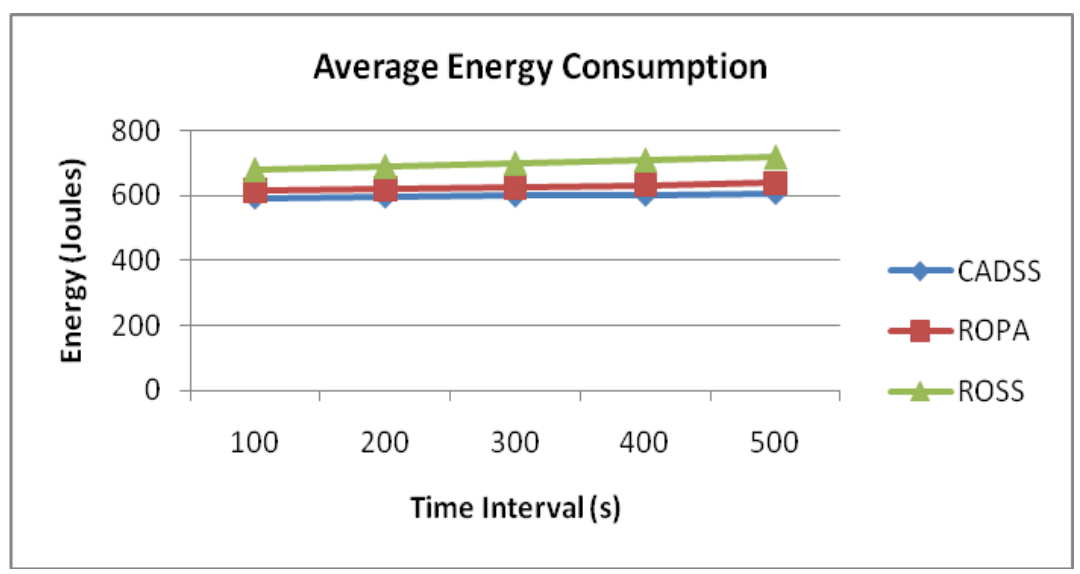


Fig 10 Energy Consumption for varying Time

Figure 10 shows the average energy consumption occurred for CADSS, ROPA and ROSS techniques when the interval is varied. The increase in interval results in increase in energy consumption. As seen from the figure, the energy consumption of CADSS increases from 592.0 to 606.5 , the energy consumption of ROPA increases from 613.6 to 638.1 and the energy consumption of ROSS increases from 680.1 to 718.3 . Hence CADSS has $4 \%$ lesser energy consumption than ROPA and 14\% lesser energy consumption than ROSS technique.

\section{Conclusion}

In this paper, we have proposed to design protocol to efficiently handle the intra-cluster collisions and to design sleep-wake up scheduling scheme for the data aggregation. In this case, the $\mathrm{CH}$ coordinates with its cluster members to transmit (append) their data packets with partially overlapping transmission times. After the $\mathrm{CH}$ finishes transmitting its packets to the courier nodes, it starts to receive incoming appended packets from its members. It then sends the packet to its parent $\mathrm{CH}$ towards the sink applying data fusion and sending the aggregated packet in TDMA period based on the receiver oriented sleep scheduling scheme. By simulation results, we have shown that the proposed technique minimizes collision and transmission delay.

\section{Declaration:}

This paper was originally prepared by me and it was not submitted to any-where for Publication

\section{Data Availability:}

The authors didn't used any thirty party data materials.

\section{References}

[1] Zhengbao Li, Zhongwen Guo, Feng Hong, Lu Hong, "An energy efficiency distributed time synchronization algorithm for underwater acoustic mobile sensor networks," Journal of Ad Hoc Networks, Vol. 11, 2013

[2] Wen-Hwa Liao, Su-Chi Kuai, Yu-Chieh Lin, “A Receiver-Initiated MAC Protocol with Packet Train Design for Underwater Acoustic Sensor Networks," Journal of Wireless Pers Communication, 2015

[3] Sai Srujana, Nehaa, Princy Mathewsa, Harigovindana, "Multi-Source Energy Harvesting System for Underwater Wireless Sensor Networks," Journal of Procedia Computer Science, Vol. 46, 2015

[4] Dongsoo Kim, Eric Noel, Wendy Tang, "WSN Communication Topology Construction with Collision Avoidance and Energy Saving," Journal of Smart Spaces and Wireless Networks, 2014

[5] Stefano Basagni, Chiara Petrioli, Roberto Petroccia, and Milica Stojanovic, "Optimized Packet Size Selection in Underwater Wireless Sensor Network Communications," IEEE Journal of Oceanic Engineering, Vol. 37, No. 3, 2012

[6] Hai-Heng, Wee-Seng Soh, Mehul Motani, "An underwater acoustic MAC protocol using reverse opportunistic packet appending," Journal of Computer Networks, Vol. 57, 2013

[7] Zuodong Wu Chen TianHongbo Jiang Wenyu Liu, "Minimum-Latency Aggregation Scheduling in Underwater Wireless Sensor Networks", in Proceedings of IEEE International Conference on Communications (ICC), pp. $1-5,2011$

[8] Tayseer Alkhdour, Uthman Baroudi, Elhadi Shakshuki, Shokri Selim, “An Optimal Cross-Layer Scheduling for Periodic WSN Applications", The 4th International Conference on Ambient Systems, Networks and Technologies (ANT 2013), Procedia Computer Science, Vol. 19, pp. 88 - 97, 2013.

[9] Lu Hong, Feng Hong, Bozhen Yang and Zhongwen Guo, "ROSS: Receiver Oriented Sleep Scheduling for Underwater Sensor Networks", In Proceedings of the Eighth ACM International Conference on Underwater Networks and Systems Article, No. 4, 2013.

[10] Kurtis Kredo, PetarDjukic and PrasantMohapatra, "STUMP: Exploiting Position Diversity in the Staggered TDMA Underwater MAC Protocol”, IEEE INFOCOM, pp. 2961 - 2965, April 2009. 
